\title{
Influencia De La Depresión En Las Reservas Proteico-Energéticas Y El Índice De Masa Corporal En Adultos Mayores De 65 Años
}

\author{
Catherine Alexandra Andrade Trujillo, $(\mathrm{MsC})$ \\ Dennys Leonardo Abril Merizalde, (MsC)
}

Docente Escuela Superior Politécnica de Chimborazo / ESPOCH Riobamba - Ecuador

Doi: 10.19044/esj.2018.v14n14p91 URL:http://dx.doi.org/10.19044/esj.2018.v14n14p91

\begin{abstract}
The aim of the study was to analyze the influence of depression on the protein-energy reserves and the Body Mass Index in ederly. A total of 65 older adults were evaluated; to identify the level of depression, the Yesavage Geriatric Depression Scale was used, the protein-energy reserves were evaluated from the arm circumference and the Body Mass Index. The elderly with higher levels of depression had lower arm circumference than those who did not show depression according to the scale $(\mathrm{p}=0.002$ and $\mathrm{p}=0.009$, men and women respectively), a similar situation occurs with the BMI ( $p=0.050$ and $\mathrm{p}=0.019)$. There is also a significant negative relationship between the Geriatric Depression Scale with the arm circumference and the BMI in women $(\mathrm{r}=-0.520, \mathrm{p}=0.002$ and $\mathrm{r}=-0.439, \mathrm{p}=0.009$ respectively). In men, this negative relationship is observed only between the Geriatric Depression Scale and the arm circumference $(r=-0.479, \mathrm{p}=0.007)$. Older adults with a higher level of depression have lower arm circumference and lower BMI than those without depression. In both sexes, there is a significant negative relationship between the Geriatric Depression Scale score and the arm circumference. In females, an inversely proportional relationship between the scale and the Body Mass Index is evidenced.
\end{abstract}

Keywords: Elderly, depression, protein-energy reserves, body mass index

\section{Resumen}

El objetivo del presente estudio fue analizar la influencia de la depresión en las reservas proteico-energéticas y el Índice de Masa Corporal (IMC) en adultos mayores de 65 años. Un total de 65 adultos mayores fueron evaluados; para identificar el nivel de depresión, se utilizó la Escala de Depresión Geriátrica de Yesavage (EDG), las reservas proteico-energéticas 
fueron evaluadas a partir de la Circunferencia del Brazo (CB) y se obtuvo el Índice de Masa Corporal. Los ancianos con mayores niveles de depresión presentaron menor circunferencia braquial que aquellos que no muestran depresión según la escala $(\mathrm{p}=0.002$ y $\mathrm{p}=0.009$; hombres y mujeres respectivamente), situación similar que ocurre con el $\operatorname{IMC}(\mathrm{p}=0.050$ y $\mathrm{p}=0.019$ ). Se evidencia, además, una relación inversamente proporcional entre el puntaje de la Escala de Depresión Geriátrica con la circunferencia braquial $\mathrm{y}$ con el IMC en mujeres ( $\mathrm{r}=-0.520 ; \mathrm{p}=0.002$ y $\mathrm{r}=-0.439 ; \mathrm{p}=0.009$ respectivamente). En los hombres esta relación negativa se observa únicamente entre el puntaje de la escala y la circunferencial braquial $\quad(\mathrm{r}=-$ $0.479 ; \mathrm{p}=0.007)$. Se concluye que los adultos mayores con mayor nivel de depresión, presentan menor circunferencia braquial y menor IMC comparado con aquellos que no presentan depresión. En ambos sexos se evidencia una relación inversamente proporcional entre el puntaje de la EDG y la $\mathrm{CB}$, es decir, a medida que aumenta el puntaje de la escala o a mayor nivel de depresión, la circunferencia braquial disminuye. En el sexo femenino se evidencia una relación inversamente proporcional entre la escala y el Índice de Masa Corporal.

Palabras clave: Adulto mayor, depresión, reservas proteico-energéticas, índice de masa corporal

\section{Introducción}

El envejecimiento constituye un proceso que incrementa las necesidades de varios nutrientes y a la vez se ve relacionado con la malnutrición debido a la disminución de la ingesta, menor apetito y en general una disminución de la calidad de vida del adulto mayor, el mismo que está sujeto a cambios tanto físicos, psíquicos, sociales como económicos (Ramón, 2001).

Varios factores de riesgo pueden hacer que el adulto mayor pierda funcionalidad y esto hace que las actividades que deba realizar precisen de la ayuda de un tercero, varios estudios de muestran que los cambios en el estilo de vida y el acceso a la educación y la promoción de la salud podrían directamente cambiar esta situación, mejorando la calidad de vida de este grupo vulnerable (Johnson, 2005).

Durante esta etapa de vida, la pérdida de masa y fuerza muscular es notable, en personas mayores de 60 años esta pérdida alcanza del 13 al 24\%, siendo superior en la población mayor de 80 años (50\% de pérdida) (Sakuma, 2015). Una pérdida de masa muscular determina una pérdida de las reservas proteico-energéticas haciendo que la funcionalidad del adulto mayor en general disminuya y que el Índice de Masa Corporal (IMC) se muestre en 
aumento debido a la marcada obesidad sarcopénica, es decir, una disminución de la masa muscular y un aumento de la masa grasa (Cox, 2016).

En cuanto a depresión, se estima a nivel nacional, que hasta el $45 \%$ de la población mayor de 65 años ha mostrado síntomas depresivos en algún momento, la etiología no se podría definirla o generalizarla debido al carácter multifactorial de la patología (Moreno, 2011). Sumado a esto, la esperanza de vida en Ecuador actualmente es de 75 años, es decir, la población está viviendo más años, pero la calidad de vida en esta etapa está siendo influenciada de manera negativa principalmente por la poca funcionalidad y los síntomas depresivos (Castro, 2011).

Pérez y colaboradores (2014), evaluaron la asociación entre la desnutrición (medida a través de la Mini Nutritional Assessment) y la depresión (medida a través de la Escala de Depresión Geriátrica de Yesavage) en adultos mayores de ambos sexos, demostrando que en el sexo femenino existe un $42 \%$ de riesgo de desarrollar desnutrición en presencia de depresión, mientras que, en el sexo masculino el riesgo es del $140 \%$.

De igual manera, Camina y colaboradores (2013), en un grupo de adultos mayores institucionalizados demuestran que a mayor grado de demencia (medido a través de escalas de tamizaje) existe una disminución de la circunferencia braquial $(\mathrm{p}=0.027)$ y del IMC $(\mathrm{p}=0.005)$. Los autores concluyen que es necesario evaluar la masa muscular y la masa grasa en esta población, ya que la medición de la circunferencia braquial es muy general y no distingue entre estos dos compartimentos corporales, por lo cual no sería posible detectar situaciones de obesidad sarcopénica, muy prevalente en este grupo de edad.

En cuanto a la depresión relacionada con el peso corporal del adulto mayor, Duran-Badillo y colaboradores (2015), establecen una correlación positiva entre estas dos variables $(\mathrm{r}=0.246 ; \mathrm{p}=0.001)$.

En torno a esta problemática, el objetivo de la presente investigación fue analizar la influencia de la depresión en las reservas proteico-energéticas y el IMC en adultos mayores de 65 años.

\section{Metodología}

El presente estudio es de tipo transversal, descriptivo, correlacional y analítico, con un total de 65 adultos mayores, pertenecientes a la Parroquia Asunción, Cantón Girón, Provincia del Azuay, elegidos por un muestreo aleatorio simple de una lista previa de 212 personas, en donde la selección de participantes se realizó con una aleatorización fija de cada 3 personas.

Para identificar el nivel de depresión en la población, se utilizó la Escala de Depresión Geriátrica de Yesavage (Geriatric Depression Scale GDS), instrumento de tamizaje que consta de 30 reactivos con respuestas dicotómicas si/no acerca de aspectos cognitivo-conductuales percibidos 
durante la semana previa a la evaluación (Martínez, 2011). Sobre un puntaje total de 30, cada ítem es valorado, como 0 si la respuesta es negativa o $1 \mathrm{si} \mathrm{la}$ respuesta es afirmativa. Un puntaje de 0 a 9 indica ausencia de depresión, 10 a 19 depresión moderada y 20 a 30 puntos depresión severa. (Yesavage, 1983).

Las reservas proteico-energéticas fueron evaluadas a partir de la circunferencia del brazo (CB), una medida antropométrica considerada como un predictor de mortalidad por la malnutrición por déficit en población adulta mayor. Se tomaron en cuenta los puntos de corte de la circunferencia braquial por sexo; siendo en hombres un déficit una $\mathrm{CB} \leq 25.4 \mathrm{~cm}$; normal $\mathrm{CB}>25.5$ $<36.8 \mathrm{~cm}$ y exceso $\geq 36.9 \mathrm{~cm}$. En el sexo femenino se considera déficit si la $\mathrm{CB}$ es $\leq 24.2 \mathrm{~cm}$, normal $>24.3-<38.8$ y exceso $\geq 38.9 \mathrm{~cm}$ (National Health and Nutrition Examination Survey, 2011).

El IMC es un indicador calculado a partir del peso y la talla del individuo, en población de adultos mayores los puntos de corte varían debido a los cambios fisiológicos que se presentan en esta etapa; $<23 \mathrm{~kg} / \mathrm{m}^{2}$ corresponde a desnutrición, IMC normal de 23 a $27,9 \mathrm{~kg} / \mathrm{m}$; de 28 a $31,9 \mathrm{~kg} / \mathrm{m}^{2}$ considerado como sobrepeso y finalmente obesidad $\geq 32 \mathrm{~kg} / \mathrm{m}^{2}$ (Ministerio de Salud Pública, 2010).

\section{Resultados}

Tabla 1. Características generales del grupo en estudio según sexo.

\begin{tabular}{ccc}
\hline Variables & $\begin{array}{c}\text { Sexo femenino } \\
(\text { Media } \pm \text { SD }) \\
(\mathrm{n}=34)\end{array}$ & $\begin{array}{c}\text { Sexo masculino } \\
(\text { Media } \pm \text { SD }) \\
(\mathrm{n}=30)\end{array}$ \\
\hline Edad (años) & $77.18 \pm 8.40$ & $77.20 \pm 8.45$ \\
EDG (puntaje) & $15.94 \pm 5.32$ & $16.17 \pm 5.29$ \\
Circunferencia braquial $(\mathrm{cm})$ & $25.50 \pm 3.21$ & $25.58 \pm 3.58$ \\
IMC $\left(\mathrm{kg} / \mathrm{m}^{2}\right)$ & $25.51 \pm 5.19$ & $25.77 \pm 5.69$ \\
\hline
\end{tabular}

$\mathrm{SD}=$ Desviación estándar; $\mathrm{EDG}=$ Escala de Depresión Geriátrica de Yesavage; $\mathrm{IMC}=$ Índice de masa corporal.

En la Tabla 1 se muestran las características generales del grupo en estudio según sexo; la media de edad es de 77 años para ambos sexos; según el puntaje de la Escala de Depresión Geriátrica de Yesavage los dos grupos presentan depresión moderada. La circunferencia braquial se encuentra dentro de los rangos de normalidad en ambos sexos, y en cuanto a la media del IMC la población en estudio presenta sobrepeso. 
Tabla 2. Análisis comparativo de la circunferencia braquial y el IMC según el nivel de depresión en el sexo femenino.

\begin{tabular}{|c|ccc|c|}
\hline \multirow{2}{*}{ VARIABLES } & \multicolumn{4}{|c|}{ Nivel de depresión, sexo femenino. Media \pm SD $(\mathrm{n}=34)$} \\
\cline { 2 - 5 } & $\begin{array}{c}\text { Sin depresión } \\
(\mathrm{n}=3)\end{array}$ & $\begin{array}{c}\text { Depresión moderada } \\
(\mathrm{n}=22)\end{array}$ & $\begin{array}{c}\text { Depresión severa } \\
(\mathrm{n}=9)\end{array}$ & $\mathrm{p}$ \\
\hline $\begin{array}{c}\text { Circunferencia } \\
\text { braquial }\end{array}$ & $28.50 \pm 5.07$ & $26.09 \pm 2.67$ & $23.06 \pm 2.50$ & $0.009 *$ \\
\hline IMC & $27.60 \pm 2.94$ & $26.88 \pm 5.60$ & $21.48 \pm 1.18$ & $0.019 *$ \\
\hline \multicolumn{5}{|c|}{ SD= Desviación estándar; EDG= Escala de Depresión Geriátrica de Yesavage; IMC= } \\
Índice de masa corporal.
\end{tabular}

En la Tabla 2 se muestra la circunferencia braquial comparada según el nivel de depresión en el sexo femenino; existen diferencias significativas entre grupos, las mujeres con mayor nivel de depresión presentan menor circunferencia braquial que aquellas que no presentan depresión (POST HOC Tukey, $\mathrm{p}=0,020)$ y aquellas que presentan depresión moderada (POST HOC Tukey, $\mathrm{p}=0,030$ ); de igual manera se evidencian diferencias significativas en el IMC, las mujeres con depresión severa presentan menor IMC que las ubicadas en el grupo de depresión moderada (POST HOC Tukey, $p=0,019$ ), siendo estas diferencias estadísticamente significativas.

Tabla 3. Análisis comparativo de la circunferencia braquial y el IMC según el nivel de depresión en el sexo masculino.

\begin{tabular}{|c|ccc|c|}
\hline \multirow{2}{*}{ VARIABLES } & \multicolumn{4}{|c|}{ Nivel de depresión EDG, sexo femenino. Media \pm SD $(\mathrm{n}=30)$} \\
\cline { 2 - 5 } & $\begin{array}{c}\text { Sin depresión } \\
(\mathrm{n}=4)\end{array}$ & $\begin{array}{c}\text { Depresión moderada } \\
(\mathrm{n}=16)\end{array}$ & $\begin{array}{c}\text { Depresión severa } \\
(\mathrm{n}=10)\end{array}$ & $\mathrm{p}$ \\
\hline $\begin{array}{c}\text { Circunferencia } \\
\text { braquial }\end{array}$ & $29.00 \pm 1.08$ & $26.50 \pm 3.16$ & $22.75 \pm 2.95$ & $0.002 *$ \\
\hline IMC & $26.17 \pm 2.88$ & $27.78 \pm 6.00$ & $22.38 \pm 4.62$ & $0.050^{*}$ \\
\hline \multicolumn{5}{|c|}{$\mathrm{SD}=$ Desviación estándar; EDG= Escala de Depresión Geriátrica de Yesavage; IMC= } \\
Índice de masa corporal.
\end{tabular}

En la Tabla 3 se muestra la circunferencia braquial comparada según el nivel de depresión en el sexo masculino; existen diferencias significativas entre grupos, los hombres con mayor nivel de depresión presentan menor circunferencia braquial comparado con aquellos que no presentan depresión (POST HOC Tukey, $\mathrm{p}=0,04$ ) y aquellos que presentan depresión moderada (POST HOC Tukey, $\mathrm{p}=0,010$ ); de igual manera se evidencian diferencias significativas en el IMC, los hombres con depresión severa presentan menor IMC que los ubicados en el grupo de depresión moderada (POST HOC Tukey, $\mathrm{p}=0,045)$, siendo estas diferencias estadísticamente significativas. 
Tabla 4. Matriz general de correlaciones por sexo.

\begin{tabular}{cccccc}
\hline \multirow{2}{*}{ Variables } & \multicolumn{2}{c}{ Sexo Femenino } & \multicolumn{2}{c}{ Sexo Masculino } \\
& & CB & IMC & CB & IMC \\
\hline \multirow{2}{*}{ EDG } & $\mathrm{r}$ & $-0.520^{* *}$ & $-0.439^{* *}$ & $-0.479^{* *}$ & -0.205 \\
& $\mathrm{p}$ & 0.002 & 0.009 & 0.007 & 0.276 \\
\hline
\end{tabular}

EDG= Escala de Depresión Geriátrica de Yesavage; $\mathbf{C B}=$ Circunferencial braquial, IMC= Índice de masa corporal, $\mathbf{r}=$ Coeficiente de correlación, $\mathbf{p}=$ significancia, $* *=$ Significativo a nivel de $\mathrm{p}=<0.01$.

La matriz general de correlaciones según el sexo se presenta en la Tabla 4, donde se evidencia una relación negativa en ambos sexos entre el puntaje de la EDG y la CB, es decir, a medida que aumenta el puntaje de la escala o a mayor nivel de depresión, la circunferencia braquial disminuye. Con estos resultados se determina que la variación de la circunferencia braquial está dada en un $27 \%$ (mujeres) y en un $22 \%$ (hombres) por el nivel de depresión. Otra relación negativa se observa entre el puntaje de la EDG y el IMC en el sexo femenino, a medida que el puntaje de la escala aumenta, el IMC disminuye, determinando que la variación del IMC está dado en un 19\% por el nivel de depresión.

\section{Discusión}

El objetivo del presente estudio fue analizar la influencia de la depresión en las reservas proteico-energéticas y el IMC en adultos mayores de 65 años, en torno a esta temática existen varios estudios realizados en población de similares características; Won y colaboradores (2016), identificaron la asociación entre la composición corporal y la función cognitiva en el adulto mayor; en donde, se determinó que las medidas antropométricas por debajo de la normalidad, entre ellas la circunferencia del brazo y el IMC, se consideran como predictores de la presencia de signos y síntomas depresivos y de ansiedad en adultos mayores de ambos sexos $(p<0.05)$, resultados que coinciden con el presente estudio en donde se evidencia que a menor circunferencia braquial e IMC, el puntaje de la Escala de Depresión Geriátrica aumenta.

De igual manera, Webb et all. (2017) concluyen que los síntomas depresivos están asociados de manera negativa con la masa grasa, sugieren que en la población anciana se debe tomar en cuenta la distribución de las reservas proteico-energéticas (masa muscular y masa grasa) ya que, si bien se observan cambios en la composición corporal por el proceso natural del envejecimiento, también se observan cambios de estos compartimentos en presencia de depresión (Luppino, 2010; Wijnhoven, 2010).

En el estudio realizado por Moreira et al. (2016), se examinaron y analizaron los cambios en los parámetros antropométricos y en la funcionalidad en el adulto mayor; los resultados sugieren que la depresión 
constituye un factor de riesgo que influencia negativamente a la funcionalidad y a la calidad de vida de esta población, al estar relacionado y ligado con los cambios en la composición corporal (reservas proteico-energéticas).

Yong-Seong y colaboradores (2017), determinaron la asociación entre la circunferencia del brazo y la presencia de demencia y depresión; los resultados fueron significativos únicamente en la población femenina para la variable demencia, lo que contrasta con el presente estudio, en donde la relación entre el nivel de depresión y la circunferencia braquial fue significativa en ambos sexos. Cabe recalcar que en el estudio de Yong-Seong, et al., la población de adultos mayores presenta un diagnóstico psiquiátrico de demencia y depresión; en el presente estudio se aplicó una encuesta de tamizaje (EDG) en la que se identifican rasgos de depresión en la población en la última semana previa a la evaluación.

En base a estos resultados, existe la controversia al saber si la depresión en la población geriátrica hace que se muestren cambios en la composición corporal, o si la composición corporal limita la funcionalidad y debido a ello es más frecuente que se presenten signos y síntomas depresivos.

\section{Conclusión}

Los adultos mayores con mayor nivel de depresión, presentan menor circunferencia braquial y menor IMC que aquellos que no presentan depresión. En ambos sexos se evidencia una relación inversamente proporcional entre el puntaje de la EDG y la CB, es decir, a medida que aumenta el puntaje de la escala o a mayor nivel de depresión, la circunferencia braquial disminuye. En el sexo femenino, además, se evidencia una relación inversamente proporcional entre la EDG y el IMC.

\section{References:}

1. Camina, M., de Mateo, B., Carreño, L., de la Cruz, S., Miján, A., Redondo, M. (2013). Cambios en la composición corporal en función del grado de demencia en un grupo de ancianos institucionalizados. Nutrición Hospitalaria. 28;3: 1093-1101. España.

2. Castro, J., Jurado, N. (2011). Situación de los ancianos en Ecuador [en línea] http://bases.bireme.br/cgibin/wxislind.exe/iah/online.

3. Cox, C. (2016). Clinical Nutrition and Aging. Sarcopenia and Muscle Metabolism. Apple Academic Press. pp272

4. Duran-Badillo, T., Martínez, M., Gutiérrez, G., Aguilar, R., GuevaraValtier, M. (2015). Peso corporal y depresión en adultos mayores de comunidad urbano marginal en México - Tamaulipas. Revista Enfermería Herediana. 8;2: 142-148. México.

5. Johnson, J. (2005). Psychosocial correlates of nutritional risk in older adults. (p66:93-95). Estados Unidos. 
6. Luppino, F., de Wit, L., Bouvy, P., Stijnen, T., Cuijpers, P., Penninx, B., et al. (2010). Overweight, obesity, and depression: a systematic review and me alysis of longitudinal studies. Archives of General Psychiatry. 67:220-229

7. Martínez, J., Onís Vilches, M., Dueñas, R. (2011). Versión española del cuestionario de Yesavage abreviado (GDS) para el despistaje de depresión en mayores de 65 años: adaptación y validación. [en línea] http://scielo.isciii.es/scielo.php?pid=S1131-

$57682002001000003 \&$ script

8. Ministerio de Salud Pública. (2010). Normas y Protocolos de Atención Integral de Salud de las y los Adultos Mayores. Quito-Ecuador.

9. Moreira, P., Correa, C., Corriente, J., Martin, L., Fortes, P., Anjos, A. (2016). Anthropometric, functional capacity, and oxidative stress changes in Brazilian community-living elderly subjects. A longitudinal study. Archives of Gerontology and Geriatrics. Brasil. 66;140-146.

10. Pérez, C., Lizárraga, D., Martínez, M. (2014). Asociación entre desnutrición y depresión en el adulto mayor. Nutrición Hospitalaria. 29;4: 901-906. México.

11. Ramón, J.M., SUBIRA, C. (2001). Prevalencia de malnutrición en población anciana Española. (117: pp766-770). Barcelona.

12. Sakuma, K., Yamaguchi, W. (2015). Current understanding of sarcopenia: Possible candidates modulating muscle mass. 467, pp213229.

13. The National Health and Nutrition Examination Survey. (2011). [en línea] https://www.cdc.gov/nchs/nhanes/index.htm

14. Yesavage, J.A., Brink, T.L., Rose, T.L. (1983). Development and validation of a geriatric depression screening scale: a preliminary report. EEUU. 17: pp37 - 49.

15. Yong-Seong, L., Sang-Dae, K., Hee-Ju, K., Sung-Wan, K., Il-Seon S., Jin-Sang Y., Jae-Min K. (2017). Associations of Upper Arm and Thigh Circumferences with Dementia and Depression in Korean Elders. Korean Neuropsychiatric Association. Korea. Pp150 - 157.

16. Webb M, Davies M, Ashra N, Bodicoat D, Brady E, Webb D, et al. (2017) The association between depressive symptoms and insulin resistance, inflammation and adiposity in men and women. PLoS ONE 12(11): e0187448.

17. Wijnhoven, H., van Bokhorst-de van der Schueren, M., Heymans, M., de Vet, H., Kruizenga, H., Twisk, J., et al. (2010). Low mid-upper arm circumference, calf circumference, and body mass index and mortality in older persons. The Journals of Gerontology. Series A, Biological Sciences and Medical Sciences. 65:1107-1114. 
18. Won, H., Abdul Manaf, Z., Mat Ludin, A., Shahar, S. (2016). Wide range of body composition measures are associated with cognitive function in community-dwelling older adults. Epidemiology, Clinical Practice and Health. 